\title{
Selected technologies to control genes and their products for experimental and clinical purposes
}

\author{
Helen K. Alexander ${ }^{1^{\star}}$, Evan P. Booy ${ }^{1,3^{\star}}$, Wenyan Xiao $^{1}$, Peyman Ezzati ${ }^{1}$, \\ Heinrich Baust ${ }^{4}$ and Marek Los ${ }^{1,2,3}$
}

${ }^{1}$ Manitoba Institute of Cell Biology, Cancer Care Manitoba, Winnipeg, Canada

${ }^{2}$ Department of Human Anatomy and Cell Science, University of Manitoba, Winnipeg, Canada

${ }^{3}$ Department of Biochemistry and Medical Genetics, University of Manitoba, Winnipeg, Canada

${ }^{4}$ Department of Radiooncology, University of Erlangen, Germany

Received: 2006.12.27, Accepted: 2007.03.15, Published online first: 2007.06.08

\begin{abstract}
"On-demand" regulation of gene expression is a powerful tool to elucidate the functions of proteins and biologically-active RNAs. We describe here three different approaches to the regulation of expression or activity of genes or proteins. Promoter-based regulation of gene expression was among the most rapidly developing techniques in the 1980s and 1990s. Here we provide basic information and also some characteristics of the metallothionein-promoter-based system, the tet-off system, Muristerone-A-regulated expression through the ecdysone response element, RheoSwitch ${ }^{\circledR}$, coumermycin/novobiocin-regulated gene expression, chemical dimerizer-based promoter activation systems, the "Dual Drug Control" system, "constitutive androstane receptor"-based regulation of gene expression, and RU486/mifepristone-driven regulation of promoter activity. A large part of the review concentrates on the principles and usage of various RNA interference techniques (RNAi: siRNA, shRNA, and miRNA-based methods). Finally, the last part of the review deals with historically the oldest, but still widely used, methods of temperature-dependent regulation of enzymatic activity or protein stability (temperature-sensitive mutants). Due to space limitations we do not describe in detail but just mention the tet-regulated systems and also fusion-protein-based regulation of protein activity, such as estrogen-receptor fusion proteins. The information provided below is aimed to assist researchers in choosing the most appropriate method for the planned development of experimental systems with regulated expression or activity of studied proteins.
\end{abstract}

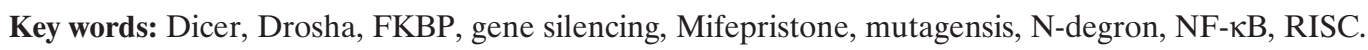

*Both authors contributed equally to this review manuscript.

Abbreviations: CAR - constitutive androstane receptor, dsRNA - double-stranded RNA, DHFR - dihydrofolate reductase, EcR - ecdysone receptor, FKBP - FK506-binding protein, FRAP - FKBP-rapamycin-associated protein, Hsp-90 - heat shock protein 90, MRE - metal-responsive elements, miRNAs - microRNAs, MCM - minichromosome maintenance, NF- $\mathrm{KB}$ nuclear factor $\mathrm{\kappa B}$, PBREM - phenobarbital-responsive enhancer module, tetR - tetracycline-dependent repressor, tTA tetracycline-controlled transactivator, tetO - tetracycline operator, TK - thymidine kinase, RXR - retinoid X receptor, RNAi - RNA interference, RISC - RNA-induced silencing complex, shRNAs - short hairpin RNAs, siRNAs - small interfering RNAs, $t d$ - temperature-inducible degron, $t s$ - temperature-sensitive, VP16 - virion protein 16 of herpes simplex virus.

Corresponding author: Marek Los, M.D. Ph.D., Manitoba Institute of Cell Biology, ON6010-675 McDermot Ave., University of Manitoba, Winnipeg, MB, R3E 0V9, Canada, tel.: +1 204 787-2294(office), -1403(lab), -4108(lab), fax: +1 204 787-2190, e-mail: losmj@cc.umanitoba.ca

\section{INTRODUCTION}

Regulated gene expression is one of the fundamental features of living organisms. Developing organisms require a dynamic and balanced regulation of gene expression in their ontogenesis, and also to respond to an ever-changing environment [18]. These processes are regulated by an orderly activation of the expressions of regulatory proteins as well as their controlled removal (degradation). Thus to better study, and intervene in, these cellular processes, a precise and selective "on-demand" turning on and off of the expressions of desired genes has been the holy grail of both cell biology and gene therapy. The large amount of information 
made available through the sequencing of the human genome as well as the genomes of other species has allowed for large-scale screening projects that aim to characterize the functions of both genes and the non-coding (or epigenetically coding) parts of genomes $[3,51]$.

The role of selected genes and their products has frequently been studied by stable overexpression of the protein of interests and not interests or its mutants. The information gained from such experiments is sometimes imprecise or, in isolated cases, simply wrong, because the cell's homeostatic mechanisms may counteract the artificially introduced changes in its metabolism. Such adaptations can mask the direct consequences of constitutively acting mutations, such as gene knock-outs and loss of function. Therefore conditional "on-demand" control of gene expression is a useful and often necessary alternative [67].

Besides the regulation of gene expression, scientists frequently use either pharmacological inhibitors, complementary peptides and peptidomimetics [42,63], or antibodies to inhibit or change the function of targeted protein(s) $[10,46,52]$. These approaches, however, also have their own problems. For example, peptides are generally unstable; peptides, peptidomimetics, and antibodies may induce an unwanted immune response during in vivo experiments; pharmacological inhibitors frequently lack high specificity; and antibodies work best on extracellular targets. Thus the most elegant way to study the function of a given protein, or biologically active RNA, is to use a regulated gene expression system. Aims to develop such systems date back to the 1960s. These "on-demand" systems to regulate the activity of selected proteins all rely on a similar concept, which generally includes a small molecule capable of modulating the binding of transcription factors to a specific promoter that drives the expression of the protein of interest. Mostly, such promoters are derived from natural ones, with the tight transcriptional regulation mechanisms that exist in vivo. These systems all have their own particular advantages and disadvantages and, although one expression system may be superior under specific circumstances, in many cases it is purely a matter of taste.

In addition to "on-demand" regulation of gene transcription, protein or even RNA activity may be regulated directly. For example by temperature-dependent regulation of a protein's stability or its function. This form of gene activity regulation is described towards the end of the review. In order to keep the review concise, we are unable to cover yet another way of regulating protein activity which is achieved by fusing the desired protein with a domain that could be regulated by a small molecule. A classical example of such regulation is based on the fusion of the estrogen receptor ligand-binding domain to a target protein [57]. In its inactive form, such a fusion protein is complexed with heat shock protein 90 (Hsp-90) and thus rendered inactive. Upon addition of $\beta$-estradiol or its analogue $\beta$-hydroxy-tamoxifen, the conformation of the estrogen receptor changes, the Hsp-90 leaves the complex, and the target protein becomes active. It is worth noting that $\mathrm{N}$ - or $\mathrm{C}$-terminal fusion of the targeted protein to the estrogen receptor often differs in the degree of leakiness and the "fold" of the achieved activation; it is therefore worthwhile to prepare both fusion proteins and then choose the most efficient system (the least leaky, but with a high fold of activation). Below we describe selected systems that allow more or less precise regulation of protein activity, either directly (for example temperature-sensitive mutants) or indirectly, by regulating the production or stability of the desired mRNAs.

\section{"ON-DEMAND" REGULATION OF GENE EXPRESSION BY INFLUENCING PROMOTER ACTIVITY: AN OVERVIEW OF CURRENTLY DEVELOPED SYSTEMS}

\section{Metallothionein promoter}

One of the earliest methods employed to control gene expression involves the use of metal-responsive promoters. This approach was experimented with by several groups in the 1980s [11, 74]. These techniques involved the use of the promoter region from the mouse metallothionein-I gene fused to the coding sequence of a gene of interest. Brinster et al. [11] discovered that transfection of mouse eggs with a plasmid containing the thymidine kinase (TK) gene under control of the mouse metallothionein-I gene allowed the induction of TK activity in the presence of cadmium. Later it was determined exactly which sequences harbored metal-responsive elements (MREs) and it was found that multiple MREs were necessary to allow zinc-induced expression [74]. These early attempts to develop regulated gene expression systems were relatively successful, but suffered from a large degree of leakiness as well as the many side effects metal ions can inflict upon cells in culture. Furthermore, manipulation of the intracellular concentration of some metals, especially zinc, may significantly affect a cell's physiology [40].

\section{Tetracycline-regulated gene expression systems}

In 1992, Gossen and Bujard [36] published a bacterial promoter-based tetracycline-controlled gene expression system that later became probably the most frequently used controlled gene expression system for mammalian cells. To date, several variants of the system have been developed and sizable literature on the subject exists (reviewed in $[7,20]$ ). For that reason, we will focus only on the most popular variant of the tetracycline-controlled expression system, namely the "tet-off"-regulated gene expression system, providing basic information on how the system is put together and how it functions.

The tetracycline-controlled "on-demand" gene expression system explores the control elements of the tetracycline-resistance operon from the tetracycline- 
-resistant strain Tn10 of E. coli, in which the transcription of the tetracycline resistance-mediated genes is negatively regulated by the tetracycline-dependent repressor (tetR) [36]. In the presence of tetracycline the tetR does not bind to its operators within the promoter region of the operon and allows transcription. By fusing the tet-repressor with the C-terminal (activating) domain of virion protein 16 of herpes simplex virus (VP16), a tetracycline-controlled transactivator (tTA) that stimulates minimal promoters fused to the tetracycline operator (tetO) has been generated. The tTA has to be constitutively expressed in the target cells. In the presence of the tetracycline the tTA cannot occupy the promoter, whereas in the absence of the tetracycline it binds to its operator sequence and strongly activates the transcription of the genes in front of it. To turn the gene expression off, tetracycline is added, and tTA is removed from the promoter and is no longer able to activate gene expression (see Fig. 1 for additional details) [36]. In our hands the system worked well, but it was still too leaky to express toxic proteins such as apoptin. Nevertheless, the system has successfully been used in a number of biological settings, both in academia and in the industry (reviewed in $[7,20]$ ).

\section{Muristerone A-regulated expression through the ecdysone response element}

Christopherson et al. [19] first demonstrated the use of a steroid receptor derived from Drosophila for the control of gene expression in mammalian cells. D. melanogaster system had a primary advantage in that the use of a foreign receptor and response element diminished the effects of endogenous receptors in mammalian cells [19]. This gene expression system is based upon the ecdysone response element of $D$. melanogaster, which is regulated during metamorphosis by the hormone $20-\mathrm{OH}$ ecdysone [70]. In 1996, No et al. [64] further optimized this system both by altering the binding specificity to a hybrid response element and by co-transfection of the ecdysone receptor $(\mathrm{EcR})$ with the retinoid $\mathrm{X}$ receptor (RXR) in order to obtain higher induction levels. The use of the RXR in conjunction with the EcR was based upon the discovery that the functional EcR is a combination of EcR dimerized with the Ultraspiracle gene product, which is the Drosophila homolog of RXR [84].

The expression system employs Muristerone A, a synthetic inducer analog of ecdysone, that efficiently induces target gene expression. The ecydysone-based gene expression system was developed by the expression of a modified EcR that was fused to the activation domain of VP16 and is capable of heterodimerization with the RXR in the presence of Muristerone A or other suitable ligand. Modifications in the $\mathrm{P}$ box of the DNA-binding domain of the EcR allow for binding to a novel response element, which is a hybrid of the glucocorticoid response element and of the type II nuclear receptors such as RXR. This not only allows for reduced basal expression of the target gene by preventing nonspecific induction, but also results in an activator that itself is not capable of binding alternative sites [64]. The advantages of this system include lower basal expression than the previously mentioned and commonly used tetracycline-based gene expression methods, higher induction (up to four orders of magnitude), the use of hydrophobic compounds that easily penetrate tissue, and faster clearance than tetracycline when used in a transgenic animal model [64]. The method was further improved upon by Saez et al. [73] in order to increase the maximal levels of induction using small molecules other than the traditionally used Muristerone A that are relatively inert in mammalian cells and have greater penetrance and induction. Although this system is slightly more cumbersome in that it requires the overexpression of two proteins, namely RXR and VpEcr, it does allow for a large degree of induction with minimal background expression and makes for a valid option for any situation where tight regulation and high induction are a must.

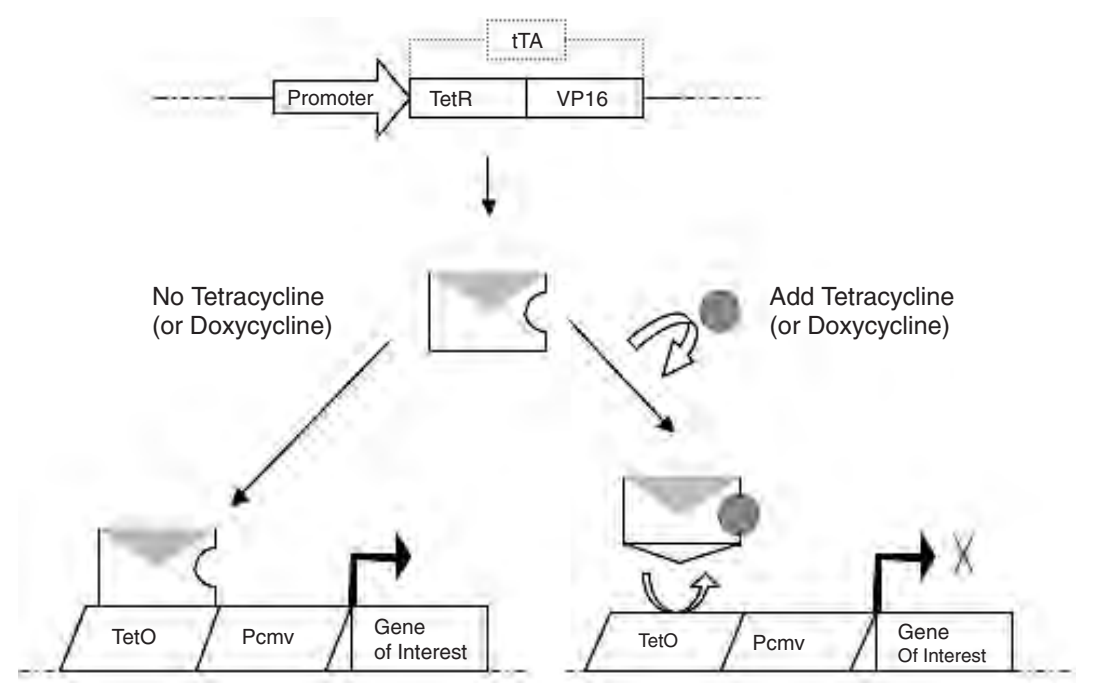

Fig. 1. The principle of "Tet-Off" regulated gene expression system. Tetracycline-controlled transcriptional regulator (tTA) was generated by fusing the DNA-binding domain of tetracycline-resistance operon (TetR) encoded in Tn10 of E. coli with the transcription activation domain of virion protein 16 of herpes simplex virus (VP16). The system requires two individual parts to be functional. First, the regulatory part, tTA, is constitutively produced under the control of a tissue specific promoter. Second the construct that contains a target gene under the control of a minimal promoter sequence of the human cytomegalovirus promoter IE (Pcmv) combined with tet operator sequences of $E$. coli (tetO). In the absence of tetracycline, tTA binds to tetO and activates Pcmv, which in turn initiates transcription of the downstream target gene. In presence of tetracycline, tTA undergoes a conformational change and dissociates from tetO, resulting in termination of transcription of the gene of interst. 
RheoSwitch ${ }^{\circledR}$ Mammalian Inducible Expression system

New England BioLabs offers a system termed the "RheoSwitch Mammalian Inducible Expression system" which is also capable of offering both strong and specific induction as well as fine-tuning of expression levels of a specific target gene. It also boasts nearly nonexistent background expression levels combined with the ability to induce expression greater than 10,000 fold. This technique, which was originally developed by Palli et al. [66] in 2003 and later improved upon by Karzenowski et al. [49] in 2005, is based upon a mechanism similar to that of the Muristerone A-inducible systems and employs a highly modified ligand-binding domain of the EcR fused to the DNA-binding domain of yeast Gal4. The activator is a fusion between the VP16-activation domain and the ligand-binding domain of RXR. The target gene is cloned downstream of five tandem repeats of the GAL4 response element. The ligand used is termed RSL1 and is a synthetic molecule that shows a complete absence of side activity in a variety of mammalian cells. Binding to the response element is dependent upon the heterodimerization of the modified RXR and EcR, which are only in an active state upon addition of the RSL1 ligand $[49,66]$. This system is commercially available and provides a greater degree of control than the original ecdysone-based expression systems developed by No et al. in 1996 [64]. It was demonstrated that the background expression is virtually nonexistent through the utilization of the system to selectively induce expression of the highly toxic diptheria toxin in insect cells [21]. The necessary plasmids and reagents are available at relatively low cost and the technique appears to be an excellent choice for the controlled expression of proteins with high toxicity.

\section{Coumermycin/novobiocin-regulated gene expression}

Another more recent method, described in 2003 by Zhao et al. [85], is the coumermycin/novobiocin-regulated gene expression system. This means of producing "on-demand" gene induction adds another layer of complexity in that it is controlled through two antibiotics, one of which is a potent inducer and another which has antagonistic function [85]. Coumermycin acts through binding of the B subunit of bacterial DNA gyrase, causing dimerization and thereby inhibiting growth [35]. The expression system exploits this function of the antibiotic by fusing the $\mathrm{B}$ subunit of bacterial DNA gyrase to the $\lambda$ repressor DNA-binding domain. To the $\mathrm{C}$ terminus of the $\mathrm{B}$ subunit of DNA gyrase, the activation domain of p65 nuclear factor (NF)- $\mathrm{KB}$ was also added, resulting in a fusion protein capable of dimerization, DNA binding, and transactivation. This transcriptional activator homodimerizes via the DNA gyrase B subunit in the presence of the antibiotic coumermycin at very low concentrations (nM range) and then binds tightly to the $\lambda$ operator. Due to high levels of basal activity, it was necessary to mutate the DNA-binding domain of the $\lambda$ repressor, as it was able to result in dimer formation in the absence of coumermycin. Novobiocin, which is an antagonist of coumermycin, efficiently switches off the expression by competitively binding DNA gyrase and reversing the homodimerization of the transactivator [85]. The primary advantage of this system is that, in addition to providing low basal expression levels and high induction, expression can rapidly be terminated via the addition of an additional antibiotic. This can be applied in systems where it is not suitable to have expression slowly return to basal levels, as can be experienced with methods that rely solely on the withdrawal of the activating molecule.

\section{Chemical dimerizers: rapamycin and analogs as examples}

Another system that uses a rather different approach is based upon the drug rapamycin, which is capable of heterodimerizing two proteins, namely FKBP (FK506-binding protein) and FRAP (FKBP-rapamycin-associated protein). This system, and a variety of its variations, use a transcriptional activation domain that is fused to FKBP and an activation domain such as that of the p65 subunit of NF- $\mathrm{kB}$ which is fused to FRAP [71]. As a result, the addition of rapamycin causes cross-linking of the DNA-binding domain to the transcriptional activation domain. Therefore, only in the presence of the drug the transcriptional activation domain is brought in proximity to the DNA-binding domain to induce transcription. In order to get around some of the negative side-effects of the drug rapamycin, mutations have been made in the FRAP protein that allow it to bind to rapamycin analogs that do not elicit the same side effects [43]. Much work has been done to optimize the system through the testing of synthetic dimerizers with promising results [2]. Furthermore, addition of the monomeric form of the dimerizer, which is only capable of binding either the DNA-binding domain fusion or the activation-domain fusion, disrupts the complex and inhibits the induced transcription, thereby allowing for a means of turning transcription off after induction [43].

\section{Dual Drug Control system}

In order to establish controlled gene induction where the expression levels are more finely tuned, a system was developed whereby expression was regulated by adding a second level of control and is termed "Dual Drug Control" [75]. The method was developed by controlling the expression of the tetracycline-controlled transactivator through a dimerization-base transactivator responsive to a small molecule. To create the first transactivator, a plasmid was developed that expresses two key proteins. The first is a fusion between the DNAbinding domain of the homeodomain protein ZFHD1 and three copies of the rapamycin-binding protein FKBP12. The second protein is a fusion between an 
activation domain that is derived from NF- $\mathrm{KB}$ and another rapamycin-binding protein, namely FRAP. These two fusion proteins will only dimerize in the presence of certain small-molecule rapamycin-related dimerizers. This dimerized transactivator is then able to bind a promoter that controls the expression of the tTA. As described earlier in this review, tTA will only bind to the tet-operator sequences in the absence of tetracycline and then, once bound, is able to induce high levels of target gene expression [36]. Overall, similar levels of induction were obtained by using a sequential or simultaneous addition of dimerizer and withdrawal of tetracycline [75]. Despite the potential benefits of a system of this nature, which may allow for enhanced levels of control and fine-tuning of expression, for most applications it will prove to require too much additional work with few benefits to be useful.

\section{Constitutive androstane receptor}

Honkakoski et al. [44] describe the creation of a gene expression system that is based on the nuclear receptor constitutive androstane receptor (CAR). CAR itself normally binds to the phenobarbital-responsive enhancer (PBREM) in response to various drugs, inducing the expression of the cytochrome P450 CYP2B genes and aiding in detoxification [45]. The basis for this system is that some steroids are able to bind and deactivate CAR [32]. In contrast to this, a range of other chemicals are able to reverse this suppression and activate CAR. By combining both suppressor and activator molecules, a gene expression system was developed that has the ability to rapidly turn expression on and off. Plasmids were developed that contain the region of the PBREM element in which the CAR-binding sites reside as well as the TK promoter driving the expression of the luciferase gene. Optimizations that included substitution of the CAR-binding sites with yeast Gal4-binding sites and fusion of the ligand-binding domain of CAR to the DNA-binding domain of GAL4 resulted in overall induction of up to 46 fold [44]. Unfortunately, this system has several drawbacks, including the high potential for nonspecific interactions of the molecules used both for suppression and activation as well as a small degree of leakiness. The resulting system is functional and provides a range of gene expression regulation using novel molecules, but it is not yet apparent whether the benefits it holds will result in mainstream use.

\section{RU486/mifepristone regulated}

An additional method for regulating gene expression is based on the discovery by Wang et al. [81] that a deletion mutant of the human progesterone receptor is incapable of binding to progesterone itself, but still retains the ability to bind antagonists such as RU486 (Mifepristone). This was exploited to develop a transactivator consisting of the mutated progesterone ligand-binding domain fused to the yeast Gal4 DNA-binding domain and the VP16-activation domain. Addition of RU486 leads to dimerization of the transactivator followed by binding to the specific promoter and strong gene induction. The concentrations of RU486 needed for strong activation are considerably lower than those needed for its antiprogesterone activity and thus it can be feasibly used in vivo [81]. This system was developed with gene therapy in mind in that it uses the Gal4 DNAbinding domain. It has no binding sites in mammalian cells, and also uses a drug that is already in use in vivo and is required at low enough concentrations to have minimal side effects and no toxicity; however, apart from this there are no major benefits of this system over many of the other methods discussed.

\section{SILENCING OF GENE EXPRESSION AT THE RNA LEVEL: SIRNA-, ShRNA-, AND mIRNA-BASED METHODS}

\section{Brief introduction to RNAi}

In addition to the several commonly used gene expression systems described in detail in previous sections of this review, there is a variety of alternative means that have been employed to control the expression of a specific gene in both cell-line and transgenic animal models. On the post-transcriptional level, using small RNA to interfere with gene expression is a very effective way to control gene activity. RNA interference (RNAi) is a ubiquitous mechanism in eukaryotic cells to suppress the expression of genes that determine fundamental cell-fate decisions of differentiation and survival [27]. RNAi could be generally defined as the silencing of gene expression by double-stranded RNA (dsRNA) which has a complementary sequence to the target gene to be silenced $[48,79]$. The sequence-specific post-transcriptional gene silencing by dsRNA is conserved in a wide range of organisms, such as plants, Neurospora, Drosophila, C. elegans, and mammals. First observed by plant biologists in the late 1980s, RNAi is related to a normal defense against viruses and the mobilization of transposable genetic elements [78].

\section{The core mechanism of RNAi}

The dsRNA trigger can be supplied exogenously, as an experimental tool, or can be derived from the genome in the form of microRNAs (miRNAs) [38]. The potent gene-silencing effect of RNAi was recognized in 1998. Studies on RNA substrate cleavage and some biochemical analyses disclosed the common underlying mechanism in different eukaryotic organisms. In RNAi, small dsRNAs processed from long dsRNAs or from transcripts that form stem-loops silence gene expression by several mechanisms. The mechanisms employed include 1) targeting mRNA for degradation, 2) preventing mRNA translation, or 3) establishing regions of silenced chromatin $[26,28]$. The two RNase machines, 
Dicer and RNA-induced silencing complex (RISC), are central players of the core RNAi pathway [31]. In the initiation step, the RNaseIII enzyme Dicer cuts the long dsRNA into small interfering RNAs (siRNAs) of approximately $21-23 \mathrm{bp}$. Then, siRNAs are loaded in the effector step. During RISC assembly, the siRNA duplex is unwound in a strand specific manner. And this singlestranded siRNA will guide the RISC to mRNA. The targeted mRNA is then cleaved by slicer inside RISC, resulting in gene silencing. Here, Dicer is also involved in the assembly of RISC [29, 30]. The remarkable natural potency of RNAi in some organisms is enhanced via an amplification step within the RNAi pathway. In the amplification step, the participation of RNA-dependent RNA polymerases for generating secondary siRNAs sustained RNAi response [31].

\section{MicroRNAs}

miRNAs are a group of small non-coding RNAs that have been identified in a variety of organisms. In eukaryotic cells, miRNA typically makes imperfect base-pair contacts with its target mRNA [77]. These small RNAs are transcribed as parts of longer molecules called pre-miRNAs, which are processed in the nucleus into hairpin RNAs of 70-100 nt by the dsRNA-specific ribonuclease Drosha [59]. Pre-miRNAs are then transported by Exportin 5 from the nucleus into the cytoplasm. In the cytoplasm, the pre-miRNA is recognized and cleaved by Dicer, in conjunction with another dsRNA binding protein into the mature miRNA. Then miRNAs cleave mRNA transcripts in a mode related to that of siRNAs. By certain computational methods it was estimated that approximately $20-30 \%$ of all human genes are targets of miRNA regulation, and there is an average of 200 targets per miRNA [23, 50, 56].

Since miRNAs are predicted to regulate a large number of animal genes, the most important characterization of the function of miRNAs is to identify the mRNA targets. Remarkably, cellular activities such as proliferation, morphogenesis, apoptosis, and differentiation are all regulated by miRNAs, and in some cases, upstream and downstream genes have been linked to the miRNAs [14]. Most miRNAs are expressed in highly tissue-specific patterns during segmentation and later stages [83]. Comprising one of the more abundant classes of gene regulatory molecules in multicellular organisms, miRNAs likely influence the output of many protein-coding genes [6]. Thus miRNAs and/or proteins involved in the processing of miRNAs are involved in various types of human disease.

The expression of miRNAs seems to be lower in cancers than in normal tissues. The reduced miRNA expression probably leads to a cancer-specific block that halts the normal development of cells and allows the tumor cells to continue to divide and grow, unlike their mature counterparts. Meanwhile, the pattern of miRNA expression varies dramatically across tumor types [59].
Remarkably, the expression pattern of this small set of miRNAs defines the cancer type better than expression data from 16,000 mRNAs. These observations could improve the diagnosis of poorly defined cancers with unknown origins, allowing better-informed choices for treatment [62]. They also promise to shed light on the regulatory circuits that malfunction during tumorigenesis. The mechanisms that regulate miRNA expression are not understood. However, techniques for finding and classifying miRNAs have been improved. The miRNA data have also been submitted to online databases. By recognizing those small molecules better, diagnosing with miRNA profiling would be possible, and using si/sh RNAs as drugs in cancer therapy would be more reasonable.

\section{siRNA and short hairpin RNAs}

RNAi has become widely used as an experimental tool to analyze the function of mammalian genes, both in vitro and in vivo, particularly in disease [60]. RNAi can be induced in mammalian cells by the introduction of synthetic siRNAs or by plasmid and viral vector systems that express double-stranded short hairpin RNAs (shRNAs) that are subsequently processed to siRNAs by the cellular machinery. Unlike synthetically produced siRNAs that are introduced to the cell by a transient transfection, shRNAs can be stably expressed in cells to down-modulate gene expression. After being expressed, shRNAs are processed into siRNAs through a mechanism similar to that of miRNAs, and then act as a kind of precursor molecule for siRNA. The major advantage of shRNA is the achievement of long-lasting gene silencing. Compared with exogenous synthetic siRNA, shRNAs expression vectors usually bring steadier and longer-term mRNA suppression in transfected cells. This long-term gene silencing ability of shRNA expression vectors provides an ample opportunity for their application in gene therapy [48].

Besides their use in functional protein studies, the shRNA coding sequences can also be used as unique identifiers, dubbed "molecular barcodes", in genomewide RNAi screens, where hundreds of constructs are analyzed in pools $[8,12]$. The approach permits the detection of both gain and loss of signal from a given shRNA, thus allowing the simultaneous identification of genes with positive effect on the phenotype of interest [12]. As for designing a shRNA insert, the same criteria applicable for the siRNA design can be employed for selecting the target in sequence followed by specific guidelines from the manufacturers that provide various shRNA expression vectors. Some important factors in determining shRNA activity include stem and loop length, sequence specificity, and the presence of overhangs [65]. In mammalian RNAi experiments, the cell type and the desired duration of the silencing effect will determine the choice of targeting molecule and delivery method. 


\section{Therapeutic usage and obstacles}

Despite the uncertainties regarding delivery and side effects, the first clinical trials with RNA therapy have already been initiated. The first siRNA drug has now entered phase I safety trials and is for age-related macular degeneration patients [31]. Current RNAi therapeutic projects include a wide range of different types of diseases, some of which will require specialized strategies for delivery of siRNA. Several groups have made some progress in anti-virus therapy, e.g. HIV and hepatitis B [16, 39, 69], and in cancer and neurodegenerative diseases $[13,17,22]$. The sequencing of the human genome has also created an urgent need to ascertain efficiently the function of novel genes and to validate targets for drug discovery. Indeed, the rapid translation of the genomic DNA sequence information into therapeutic strategies for many common maladies, particularly infectious, cardiovascular, neoplastic, and neurological diseases, would be highly desirable. siRNAs may be the best tools for target validation in biomedical research today because of their exquisite specificity, efficiency, and endurance of gene-specific silencing. siRNAs are probably also suitable for the design of novel gene-specific therapeutics by directly targeting the mRNAs of disease-related genes [79]. Though the application of RNAi as a therapeutic modality undoubtedly is very promising, using siRNA as small-molecule drugs just began three years ago. There are many obstacles to deal with, such as in vivo stability and resistance to serum RNase, effective delivery to the tissue(s) of interest, and decreased nonspecific and immunostimulatory effects.

\section{TEMPERATURE-DEPENDENT REGULATION OF PROTEIN ACTIVITY: HEAT-SENSITIVE MUTANTS}

Temperature-sensitive (ts) mutants are among the most common conditional mutants. The mutated proteins are usually sensitive to heat: at lower (permissive) temperatures, the gene produces normal amounts of a functional product, as in the wild-type; at higher (restrictive or non-permissive) temperatures, the gene loses its normal functionality and/or does not generate significant amounts of active product [15, 25]. Conditional mutants are valuable tools in the investigation of a protein's role and of the physiological effects of inactivating a gene, both in vivo and in cell culture $[15$, 25]. Adaptations can mask the direct consequences of constitutively acting mutations, such as gene knock-outs and loss of function; therefore, conditional control is a useful alternative [67]. Temperature-sensitive mutants have the particular advantages of ease of use (no change of media required for induction), reversibility, quick response time, and flexibility of application in virtually any tissue or stage of growth [15, 24]. They have been identified in a wide variety of systems, including viruses, bacteria, Paramecium, Neurospora, yeast, Arabidopsis, Drosophila, and mammalian cells $[67,76]$. However, $t$ s mutations cannot be studied within homeothermic organisms [80]. In addition, leaky expression at non-permissive temperatures is a common problem among $t s$ mutations [25, 68].

The classical approach to generating $t s$ mutants uses random mutagenesis (usually with a chemical mutagen), followed by the labor-intensive and not always successful process of screening progeny [15]. Large genomes, a lack of simple screening methods, a small number of progeny, or lengthy generation times can render this approach very difficult or ineffective [80]. Nevertheless, random mutagenesis has been in use for decades; for example by Suzuki et al. in 1967 [76], with the goal of generating Drosophila melanogaster ts mutants for future use in genetic analysis. Male Drosophila were treated with ethyl methanesulfonate, mitomycin c, or $\gamma$-irradiation. The progeny of subsequent crosses were screened for temperature-dependent lethal mutations. More recently, Parrini and Mayer [67] used a modern technique of random in vitro mutagenesis to investigate the interaction between the SH3-2 domain of Nck, an adaptor protein, and the N-terminal of Pak1 kinase. SH3 domains are frequently involved in signal transduction; conditional control of these interactions could help to elucidate the pathways. PCR with decreased Taq polymerase fidelity was used to generate mutations in Nck SH3-2 DNA, followed by in vivo recombination of PCR products into pACT2 vector in yeast that also expressed N-terminal Pak1. Yeast two-hybrid screening distinguished clones showing temperature-dependent Nck/Pak1 interactions. Screening of $3 \times 10^{4}$ colonies identified 12 unique mutants, four of which showed temperature sensitivity in mammalian (293T) cells.

To reduce the labor required by random mutagenesis, site-directed mutagenesis has been employed to generate $t s$ mutants. Varadarajan et al. [80] developed one such method, relying on the amino-acid sequence of the protein of interest (which must be globular). Based on calculations of hydrophobicity, probable buried residues are identified and targeted for substitution to destabilize the protein. Specific amino acids most likely to induce temperature sensitivity are introduced at these positions. Given this relatively small number of candidates, site-directed mutagenesis at each position followed by appropriate screening methods is a practical approach with a high success rate. The authors used this method to predict accurately several known ts mutants in viral and bacterial proteins [80]. This approach was honed by Chakshusmathi et al. [15], who generated previously unknown ts mutants of the E. coli cytotoxin CcdB and the yeast transcriptional activator Gal4. The latter have potentially valuable applications through the use of the upstream-activating-sequence-Gal4 system to conditionally control genes.

Another approach to site-directed mutagenesis is based on comparison with known ts mutants in a similar protein. For example, Parrini and Mayer [67] analyzed 
the $t s$ Nck SH3-2 mutants by DNA sequencing to identify the relevant substitutions. Similar substitutions were made at corresponding positions in the SH3 domain of Crk-1, another adaptor protein, accelerating the production of a new $t s$ mutant. Similarly, Lee et al. [54] selected substitutions that resulted in known $t s$ mutants in $E$. coli dnaA and made the same modifications at corresponding sites of Streptomyces lividans and Streptomyces coelicolor dnaA to generate previously unknown $t s$ mutants in these bacteria.

An inherent drawback of the mutagenesis approach is that it may be difficult or impossible to produce a $t s$ mutation in the gene of interest [25, 68]. Dohmen et al. [25] designed an alternative method in Saccharomyces cerevisiae that constructs mutants by fusion to a heatinducible degron, rather than requiring a $t s$ mutation within the given gene. The wild-type N-terminal Val in mouse dihydrofolate reductase (DHFR) is substituted by Arg, a destabilizing residue in the $\mathrm{N}$-end rule (the correspondence between the in vivo stability of a protein and its particular N-terminal residue). An appropriate N-terminal residue and a specific internal Lys residue to act as a ubiquitination site constitute an N-degron, a type of intracellular degradation signal. The group identified a $t s$ allele of Arg-DHFR that is stable at $23 \mathrm{C}$ but not at $37 \mathrm{C}$, due to partial unfolding of the protein that increases the accessibility of the N-terminal Arg and/or internal Lys and thus activates the N-degron. A $t s$ Arg-DHFR construct (which also contains an hemagglutinin epitope to allow immunoprecipitation and detection by Western blot) can be fused to the N-terminal of a protein of interest; the processive nature of $\mathrm{N}$-end rule proteolysis implies that the entire fusion is thermolabile. This method was demonstrated with S. cerevisiae Ura3 and Cdc28 fusions; in both cases, the protein was long-lived at $23 \mathrm{C}$, but short-lived at $37 \mathrm{C}$, resulting in a $t s$ phenotype. The authors refer to this type of $t s$ mutant as a temperature-inducible degron $(t d)$ mutant [25].

Despite some successes with direct use of this method [24], protein degradation at the non-permissive temperature is not always satisfactory. To investigate the roles of minichromosome maintenance (MCM) proteins in eukaryotic DNA replication, Labib et al. [53] attempted to use this method to generate $t d$ mutants of MCMs. At $37 \mathrm{C}$ the proteins were degraded slowly and retained some activity. To solve this problem, the group used the GAL1-10 promoter to control the expression of UBR1, which encodes $\mathrm{N}$-degron recognition factor. Upon induction with galactose of high GAL-UBR1 expression, MCM proteins were efficiently degraded at $37 \mathrm{C}$. Kanemaki et al. [47] have extended this method for efficient generation of multiple degron fusions in a single yeast strain using a PCR-based procedure.

Td mutants hold a key advantage over other conditional mutants: the speed at which protein levels are depleted and the corresponding phenotype is conferred upon heat induction. In contrast, repressing transcription via conditional promoter control commonly results in phenotypic lag, with possible secondary cellular effects due to a gradual decrease in protein levels [24,
47]. It is often crucial to avoid this problem to elucidate the precise functions of proteins, such as those involved in the cell cycle [47]. Conditional promoters also result in unnatural levels of gene expression, whereas a $t d$ protein can theoretically be expressed from its normal promoter [24]. Compared with other ts proteins, which often show diminished activity at the permissive temperature, $t d$ fusions usually have the benefit of being functionally normal under this condition [25]. However, the $t d$ fusion method also has its limitations; thus, other approaches to generating $t s$ mutants have not been rendered obsolete. The method is applicable only to proteins that accept $\mathrm{N}$-terminal extension and those that exist in the cellular compartments where the $\mathrm{N}$-end rule pathway is known to function (the nucleus and cytosol and not, for instance, in the secretory pathway) [24, 25]. A fusion protein may not function identically to the wild-type, even at permissive temperatures [80]. Although the heat-inducible N-degron method has been extended to Schizosaccharomyces pombe [68], little usage has yet been attempted outside of yeast. Levy et al. [55] investigated the expression and degradation of Arg-DHFR ${ }^{\text {ts }}$ and Met-DHFR ${ }^{\text {ts }}$ in mouse L cells, but did not construct $t d$ fusion proteins in these cells. Further developments will be required in order to take full advantage of the fusion method in higher eukaryotes $[47,80]$.

To extend its applications, the N-degron method could also be combined with other types of conditional control. For example, methotrexate inhibition of Arg-DHFR ${ }^{\text {ts }}$ degradation at non-permissive temperatures could potentially be used to develop conditional mutants in homeothermic organisms [55]. In addition, similar $t d$ fusion methods could be developed with other degrons, allowing control of proteins inaccessible to the $\mathrm{N}$-end rule mechanism [24].

\section{CLOSING REMARKS}

The three distinct sets of methods outlined above that allow for the control of gene/protein activity all fulfill different demands and have different, yet overlapping, fields of application. Certainly the siRNA technology is now the most dynamically developing field of gene silencing and it will likely have the strongest impact in medicine, at least in the near future. Not long after the mechanism was elucidated, siRNAs have become a powerful laboratory tool for functional research and potential drug development for different kinds of diseases. As the miRNAs seem to be distinct for different types of cancer, studies of the miRNA patterns of expression in cancer cells will provide a new and powerful diagnostic tool and may lead to a better understanding of the discrete changes in cancer cells that drive uncontrolled proliferation and resistance to therapy.

Despite over 40 years of research and development, the currently available "on-demand" gene regulation systems are still not optimal. The leakiness of the used 
promoter regulation systems still make it impossible to work with particularly toxic proteins, such as apoptin [61]. The answer may come with the development of "two-level" regulatory systems, where both the promoter and the mRNA stability of the protein of interest will be controlled. Thus, in the "off"state the promoter of the target gene will be switched off, and the expression of shRNA will be activated to target the leaky expression of the protein of interest. In the "on" state the protein of interest would be expressed, but the shRNA that targets its mRNA will be switched off.

Beside therapeutic approaches that focus on the sole application of gene regulation, methods combining two or more therapeutic principles are being developed. As we learn more and more about the regulation of gene expression during therapeutic interventions $[37,58]$, it is becoming possible to put the expression of therapeutic proteins under the control of promoters that will become active during a standard therapy. For instance, the EGR1 promoter has been found to be activated by ROS produced after ionizing radiation exposure. Altering the number and core sequences of the CArG elements of the EGR1 promoter can affect radioinducibility. Thus, the EGR1 promoter can be used to induce the expression of proapoptotic or suicide genes as, for example, Bax or HSVtk/GCV [1, 82].

Despite the obvious deficiencies outlined in this review, "on-demand" gene regulation mechanisms have been playing a crucial role in the elucidation of biologic processes, and they will likely finally find their way to the clinic. Current cancer therapies either more or less indiscriminately target rapidly proliferating cells, or they aim at molecules overexpressed by cancer cells $[4,9,41$, $46,52,63,72]$. The "on-demand" regulated gene expression systems provide almost countless possibilities to target, in a regulated manner, the expression of proteins that, for example, drive the uncontrolled proliferation of cancer cells or their resistance towards classical therapies. When combined with rapid in vivo cell-death detection methods, such as the detection of serum cytochrome c [5, 33, 34], these new therapeutic approaches may significantly improve the outcome of cancer treatment.

Acknowledgment: M. Los thankfully acknowledges the financial support by the CFI-Canada Research Chair program and the PCRFC, CCMF, MMSF, MHRC, and CIHR foundations. E. P. Booy thankfully acknowledges the support by the fellowships funded by the CIHR and by the University of Manitoba.

\section{REFERENCES}

1. Ahmed M. M. (2004): Regulation of radiation-induced apoptosis by early growth response- 1 gene in solid tumors. Curr. Cancer Drug Targets, 4, 43-52.

2. Amara J. F., Clackson T., Rivera V. M., Guo T., Keenan T., Natesan S., Pollock R., Yang W., Courage N. L., Holt D. A. and Gilman M. (1997): A versatile synthetic dimerizer for the regulation of protein-protein interactions. Proc. Natl. Acad. Sci. USA, 94, 10618-10623.
3. Anderson J. E., Hansen L. L., Mooren F. C., Post M., Hug H., Zuse A. and Los M. (2006): Methods and biomarkers for the diagnosis and prognosis of cancer and other diseases: towards personalized medicine. Drug Resist. Updat., 9, 198-210.

4. Banerji S. and Los M. (2006): Important differences between topoisomerase-I and -II targeting agents. Cancer Biol. Ther., 5, 965-966.

5. Barczyk K., Kreuter M., Pryjma J., Booy E. P., Maddika S., Ghavami S., Berdel W. E., Roth J. and Los M. (2005): Serum cytochrome $\mathrm{c}$ indicates in vivo-apoptosis and it can serve as a prognostic marker during cancer therapy. Int. J. Cancer, 114, 167-173.

6. Bartel D. P. (2004): MicroRNAs: genomics, biogenesis, mechanism, and function. Cell, 116, 281-297.

7. Berens C. and Hillen W. (2003): Gene regulation by tetracyclines. Constraints of resistance regulation in bacteria shape TetR for application in eukaryotes. Eur. J. Biochem., 270, 3109-3121.

8. Berns K., Hijmans E. M., Mullenders J., Brummelkamp T. R., Velds A., Heimerikx M., Kerkhoven R. M., Madiredjo M., Nijkamp W., Weigelt B., Agami R., Ge W., Cavet G., Linsley P. S., Beijersbergen R. L. and Bernards R. (2004): A large-scale RNAi screen in human cells identifies new components of the p53 pathway. Nature, 428, 431-437.

9. Blagosklonny M. V. and Darzynkiewicz Z. (2005): Strategies of protection of normal cells during chemo- and radio-therapy based on modulation of cell cycle and apoptotic pathways. In Los M. and Gibson S. B. (eds.): Apoptotic pathways as target for novel therapies in cancer and other diseases. Springer Akademic Press, New York, 357-391.

10. Booy E. P., Johar D., Maddika S., Pirzada H., Sahib M. M., Gehrke I., Loewen S. D., Louis S. D., Kadkhoda K., Mowat M. and Los M. (2006): Monoclonal and bispecific antibodies as novel therapeutics. Arch. Immunol. Ther. Exp., 54, 1-17.

11. Brinster R. L., Chen H. Y., Warren R., Sarthy A. and Palmiter R. D. (1982): Regulation of metallothionein thymidine kinase fusion plasmids injected into mouse eggs. Nature, 296, 39-42.

12. Brummelkamp T. R. and Bernards R. (2003): New tools for functional mammalian cancer genetics. Nat. Rev. Cancer, 3, 781-789.

13. Brummelkamp T. R., Bernards R. and Agami R. (2002): Stable suppression of tumorigenicity by virus-mediated RNA interference. Cancer Cell, 2, 243-247.

14. Carthew R. W. (2006): Gene regulation by microRNAs. Curr. Opin. Genet. Dev., 16, 203-208.

15. Chakshusmathi G., Mondal K., Lakshmi G. S., Singh G., Roy A., Ch R. B., Madhusudhanan S. and Varadarajan R. (2004): Design of temperature-sensitive mutants solely from amino acid sequence. Proc. Natl. Acad. Sci. USA, 101, 7925-7930.

16. Check E. (2005): Pioneering HIV treatment would use interference and gene therapy. Nature, 437, 601.

17. Chen J., Wall N. R., Kocher K., Duclos N., Fabbro D., Neuberg D., Griffin J. D., Shi Y. and Gilliland D. G. (2004): Stable expression of small interfering RNA sensitizes TEL-PDGFbetaR to inhibition with imatinib or rapamycin. J. Clin. Invest., 113, 1784-1791.

18. Cherlonneix L. (2004): From life without death to permanent delaying of death in life (De la vie sans mort à la Vie en suspens). Rev. Hist. Epistemologie Sci. Vie., 11, 139-161. 
19. Christopherson K. S., Mark M. R., Bajaj V. and Godowski P. J. (1992): Ecdysteroid-dependent regulation of genes in mammalian cells by a Drosophila ecdysone receptor and chimeric transactivators. Proc. Natl. Acad. Sci. USA, 89, 6314-6318.

20. Corbel S. Y. and Rossi F. M. (2002): Latest developments and in vivo use of the Tet system: ex vivo and in vivo delivery of tetracycline-regulated genes. Curr. Opin. Biotechnol., 13, 448-452.

21. Dai X., Willis L. G., Palli S. R. and Theilmann D. A. (2005): Tight transcriptional regulation of foreign genes in insect cells using an ecdysone receptor-based inducible system. Protein Expr. Purif., 42, 236-245.

22. Deininger M., Buchdunger E. and Druker B. J. (2005): The development of imatinib as a therapeutic agent for chronic myeloid leukemia. Blood, 105, 2640-2653.

23. Di Leva G., Calin G. A. and Croce C. M. (2006): MicroRNAs: fundamental facts and involvement in human diseases. Birth Defects Res. C Embryo Today, 78, 180-189.

24. Dohmen R. J. (2006): Inducible degron and its application to creating conditional mutants. Methods Mol. Biol., 313, 145-159.

25. Dohmen R. J., Wu P. and Varshavsky A. (1994): Heat-inducible degron: a method for constructing temperature-sensitive mutants. Science, 263, 1273-1276.

26. Dykxhoorn D. M. and Lieberman J. (2005): The silent revolution: RNA interference as basic biology, research tool, and therapeutic. Annu. Rev. Med., 56, 401-423.

27. Dykxhoorn D. M. and Lieberman J. (2006): Running interference: prospects and obstacles to using small interfering RNAs as small molecule drugs. Annu. Rev. Biomed. Eng., 8, 377-402.

28. Dykxhoorn D. M., Novina C. D. and Sharp P. A. (2003): Killing the messenger: short RNAs that silence gene expression. Nat. Rev. Mol. Cell Biol., 4, 457-467.

29. Elbashir S. M., Harborth J., Lendeckel W., Yalcin A., Weber K. and Tuschl T. (2001): Duplexes of 21-nucleotide RNAs mediate RNA interference in cultured mammalian cells. Nature, 411, 494-498.

30. Elbashir S. M., Martinez J., Patkaniowska A., Lendeckel W. and Tuschl T. (2001): Functional anatomy of siRNAs for mediating efficient RNAi in Drosophila melanogaster embryo lysate. EMBO J., 20, 6877-6888.

31. Fjose A. and Drivenes O. (2006): RNAi and microRNAs: from animal models to disease therapy. Birth Defects Res. C Embryo Today, 78, 150-171.

32. Forman B. M., Tzameli I., Choi H. S., Chen J., Simha D., Seol W., Evans R. M. and Moore D. D. (1998): Androstane metabolites bind to and deactivate the nuclear receptor CAR-beta. Nature, 395, 612-615.

33. Ghavami S., Barczyk K., Maddika S., Vogl T., Steinmüller L., Pour-Jafari H., Evans J. A. and Los M. (2005): Monitoring of programmed cell death in vivo and in vitro new and old methods of cancer therapy assessment. In Los M. and Gibson S. B. (eds.): Apoptotic pathways as target for novel therapies in cancer and other diseases. Springer Academic Press, New York, 323-341.

34. Ghavami S., Hashemi M., Kadkhoda K., Alavian S. M., Bay G. H. and Los M. (2005): Apoptosis in liver diseases detection and therapeutic applications. Med. Sci. Monit., 11, RA337-345.

35. Gilbert E. J. and Maxwell A. (1994): The 24 kDa N-terminal sub-domain of the DNA gyrase B protein binds coumarin drugs. Mol. Microbiol., 12, 365-373.
36. Gossen M. and Bujard H. (1992): Tight control of gene expression in mammalian cells by tetracycline-responsive promoters. Proc. Natl. Acad. Sci. USA, 89, 5547-5551.

37. Grote J., Konig S., Ackermann D., Sopalla C., Benedyk M., Los M. and Kerkhoff C. (2006): Identification of poly(ADP-ribose)polymerase- 1 and $\mathrm{Ku} 70 / \mathrm{Ku} 80$ as transcriptional regulator of S100A9 gene expression. BMC Mol. Biol., 7, 48.

38. Hammond S. M. (2005): Dicing and slicing: the core machinery of the RNA interference pathway. FEBS Lett., 579, 5822-5829.

39. Hannon G. J. and Rossi J. J. (2004): Unlocking the potential of the human genome with RNA interference. Nature, 431, 371-378.

40. Hashemi M., Ghavami S., Eshraghi M., Booy E. P. and Los M. (2007): Cytotoxic effects of intra- and extracellular zinc chelation on human breast cancer cells. Eur. J. Pharmacol., 557, 9-19.

41. Hashemi M. and Kroczak T. J. (2005): Apoptosis and autoimmune disease. Curr. Med. Chem. - Anti-Inflammatory \& Anti-Allergy Agents, 4.

42. Hauff K., Zamzow C., Law W. J., de Melo J., Kennedy K. and Los M. (2005): Peptide-based approaches to treat asthma, arthritis, other autoimmune diseases and pathologies of the central nervous system. Arch. Immunol. Ther. Exp., 53, 308-320.

43. Ho S. N., Biggar S. R., Spencer D. M., Schreiber S. L. and Crabtree G. R. (1996): Dimeric ligands define a role for transcriptional activation domains in reinitiation. Nature, 382, 822-826.

44. Honkakoski P., Jaaskelainen I., Kortelahti M. and Urtti A. (2001): A novel drug-regulated gene expression system based on the nuclear receptor constitutive androstane receptor (CAR). Pharm. Res., 18, 146-150.

45. Honkakoski P., Zelko I., Sueyoshi T. and Negishi M. (1998): The nuclear orphan receptor CAR-retinoid X receptor heterodimer activates the phenobarbital-responsive enhancer module of the CYP2B gene. Mol. Cell Biol., 18, 5652-5658.

46. Johnston J. B., Navaratnam S., Pitz M. W., Maniate J. M., Wiechec E., Baust H., Gingerich J., Skliris G. P., Murphy L. C. and Los M. (2006): Targeting the EGFR pathway for cancer therapy. Curr. Med. Chem., 13, 3483-3492.

47. Kanemaki M., Sanchez-Diaz A., Gambus A. and Labib K. (2003): Functional proteomic identification of DNA replication proteins by induced proteolysis in vivo. Nature, $\mathbf{4 2 3}$, 720-724.

48. Karkare S., Daniel S. and Bhatnagar D. (2004): RNA interference silencing the transcriptional message: aspects and applications. Appl. Biochem. Biotechnol., 119, 1-12.

49. Karzenowski D., Potter D. W. and Padidam M. (2005): Inducible control of transgene expression with ecdysone receptor: gene switches with high sensitivity, robust expression, and reduced size. Biotechniques, 39, 191-192, 194, 196 passim.

50. Krek A., Grun D., Poy M. N., Wolf R., Rosenberg L., Epstein E. J., MacMenamin P., da Piedade I., Gunsalus K. C., Stoffel M. and Rajewsky N. (2005): Combinatorial microRNA target predictions. Nat. Genet., 37, 495-500.

51. Kroczak T. J., Baran J., Pryjma J. S., M., Rashedi I., Hernandez E., Alberti E. M., S. and Los M. (2006): The emerging importance of DNA mapping and other comprehensive screening techniques as tools to identify new drug targets and as a mean of (cancer) therapy personalization. Expert Opin. Ther. Targets, 10, 289-302. 
52. Krzemieniecki K., Szpyt E., Rashedi I., Gawron K. and Los M. (2006): targeting of solid tumors and blood malignancies by antibody-based therapies. Centr. Eur. J. Biol., 1, 167-182.

53. Labib K., Tercero J. A. and Diffley J. F. (2000): Uninterrupted MCM2-7 function required for DNA replication fork progression. Science, 288, 1643-1647.

54. Lee L. F., Yeh S. H. and Chen C. W. (2002): Construction and synchronization of dnaA temperature-sensitive mutants of Streptomyces. J. Bacteriol., 184, 1214-1218.

55. Levy F., Johnston J. A. and Varshavsky A. (1999): Analysis of a conditional degradation signal in yeast and mammalian cells. Eur. J. Biochem., 259, 244-252.

56. Lewis B. P., Burge C. B. and Bartel D. P. (2005): Conserved seed pairing, often flanked by adenosines, indicates that thousands of human genes are microRNA targets. Cell, 120, 15-20.

57. Los M., Khazaie K., Schulze Osthoff K., Baeuerle P. A., Schirrmacher V. and Chlichlia K. (1998): Human T cell leukemia virus-I (HTLV-I) Tax-mediated apoptosis in activated $\mathrm{T}$ cells requires an enhanced intracellular prooxidant state. J. Immunol., 161, 3050-3055.

58. Los M., Mozoluk M., Ferrari D., Stepczynska A., Stroh C., Renz A., Herceg Z., Wang Z.-Q. and Schulze-Osthoff K. (2002): Activation and caspase-mediated inhibition of PARP: a molecular switch between fibroblast necrosis and apoptosis in death receptor signaling. Mol. Biol. Cell, 13, 978-988.

59. Lu J., Getz G., Miska E. A., Alvarez-Saavedra E., Lamb J., Peck D., Sweet-Cordero A., Ebert B. L., Mak R. H., Ferrando A. A., Downing J. R., Jacks T., Horvitz H. R. and Golub T. R. (2005): MicroRNA expression profiles classify human cancers. Nature, 435, 834-838.

60. Maddika S., Booy E. P., Johar D., Gibson S. B., Ghavami S. and Los M. (2005): Cancer-specific toxicity of apoptin is independent of death receptors but involves the loss of mitochondrial membrane potential and the release of mitochondrial cell death mediators by a Nur77-dependent pathway. J. Cell Sci., 118, 4485-4493.

61. Maddika S., Mendoza F. J., Hauff K., Zamzow C. R., Paranjothy T. and Los M. (2006): Cancer-selective therapy of the future: apoptin and its mechanism of action. Cancer Biol. Ther., 5, 10-19.

62. Meltzer P. S. (2005): Cancer genomics: small RNAs with big impacts. Nature, 435, 745-746.

63. Mendoza F. J., Espino P. S., Cann K. L., Bristow N., McCrea K. and Los M. (2005): Anti-tumor chemotherapy utilizing peptide-based approaches-apoptotic pathways, kinases, and proteasome as targets. Arch. Immunol. Ther. Exp., 53, 47-60.

64. No D., Yao T. P. and Evans R. M. (1996): Ecdysoneinducible gene expression in mammalian cells and transgenic mice. Proc. Natl. Acad. Sci. USA, 93, 3346-3351.

65. Paddison P. J., Caudy A. A., Bernstein E., Hannon G. J. and Conklin D. S. (2002): Short hairpin RNAs (shRNAs) induce sequence-specific silencing in mammalian cells. Genes Dev., 16, 948-958.

66. Palli S. R., Kapitskaya M. Z., Kumar M. B. and Cress D. E. (2003): Improved ecdysone receptor-based inducible gene regulation system. Eur. J. Biochem., 270, 1308-1315.

67. Parrini M. C. and Mayer B. J. (1999): Engineering temperature-sensitive SH3 domains. Chem. Biol., 6, 679-687.

68. Rajagopalan S., Liling Z., Liu J. and Balasubramanian M. (2004): The N-degron approach to create temperature-sensitive mutants in Schizosaccharomyces pombe. Methods, 33, 206-212.
69. Rendall G. (2005): Progress toward the therapy of hepatitis with RNAi. Hepatology, 41, 1220-1222.

70. Riddiford L. M. (1993): The Development of Drosophila melangaster. Plainview: Cold Spring Harbor Lab Press, vol. 2 .

71. Rivera V. M., Clackson T., Natesan S., Pollock R., Amara J. F., Keenan T., Magari S. R., Phillips T., Courage N. L., Cerasoli F., Jr., Holt D. A. and Gilman M. (1996): A humanized system for pharmacologic control of gene expression. Nat. Med., 2, 1028-1032.

72. Ross J. S., Schenkein D. P., Pietrusko R., Rolfe M., Linette G. P., Stec J., Stagliano N. E., Ginsburg G. S., Symmans W. F., Pusztai L. and Hortobagyi G. N. (2004): Targeted therapies for cancer 2004. Am. J. Clin. Pathol., 122, 598-609.

73. Saez E., Nelson M. C., Eshelman B., Banayo E., Koder A., Cho G. J. and Evans R. M. (2000): Identification of ligands and coligands for the ecdysone-regulated gene switch. Proc. Natl. Acad. Sci. USA, 97, 14512-14517.

74. Searle P. F., Stuart G. W. and Palmiter R. D. (1985): Building a metal-responsive promoter with synthetic regulatory elements. Mol. Cell Biol., 5, 1480-1489.

75. Sudomoina M., Latypova E., Favorova O. O., Golemis E. A. and Serebriiskii I. G. (2004): A gene expression system offering multiple levels of regulation: the Dual Drug Control (DDC) system. BMC Biotechnol., 4, 9.

76. Suzuki D. T., Piternick L. K., Hayashi S., Tarasoff M., Baillie D. and Erasmus U. (1967): Temperature-sensitive mutations in Drosophila melanogaster. I. Relative frequencies among gamma-ray and chemically induced sex-linked recessive lethals and semilethals. Proc. Natl. Acad. Sci. USA, 57, 907-912.

77. Tang G. (2005): siRNA and miRNA: an insight into RISCs. Trends Biochem. Sci., 30, 106-114.

78. Tijsterman M., Ketting R. F. and Plasterk R. H. (2002): The genetics of RNA silencing. Annu. Rev. Genet., 36, 489-519.

79. Tuschl T. and Borkhardt A. (2002): Small interfering RNAs: a revolutionary tool for the analysis of gene function and gene therapy. Mol. Interv., 2, 158-167.

80. Varadarajan R., Nagarajaram H. A. and Ramakrishnan C. (1996): A procedure for the prediction of temperature-sensitive mutants of a globular protein based solely on the amino acid sequence. Proc. Natl. Acad. Sci. USA, 93, 13908-13913.

81. Wang Y., O’Malley B. W. Jr., Tsai S. Y. and O’Malley B. W. (1994): A regulatory system for use in gene transfer. Proc. Natl. Acad. Sci. USA, 91, 8180-8184.

82. Weichselbaum R. R., Kufe D. W., Hellman S., Rasmussen H. S., King C. R., Fischer P. H. and Mauceri H. J. (2002): Radiation-induced tumour necrosis factor-alpha expression: clinical application of transcriptional and physical targeting of gene therapy. Lancet Oncol., 3, 665-671.

83. Wienholds E., Kloosterman W. P., Miska E., AlvarezSaavedra E., Berezikov E., de Bruijn E., Horvitz H. R., Kauppinen S. and Plasterk R. H. (2005): MicroRNA expression in zebrafish embryonic development. Science, 309, 310-311.

84. Yao T. P., Forman B. M., Jiang Z., Cherbas L., Chen J. D., McKeown M., Cherbas P. and Evans R. M. (1993): Functional ecdysone receptor is the product of EcR and Ultraspiracle genes. Nature, 366, 476-479.

85. Zhao H. F., Boyd J., Jolicoeur N. and Shen S. H. (2003): A coumermycin/novobiocin-regulated gene expression system. Hum. Gene Ther., 14, 1619-1629. 